TITLE:

\title{
Multiple-division subgrid construction for space-time finite integration electromagnetic field computation
}

\section{$\operatorname{AUTHOR}(\mathrm{S})$ :}

Sakata, Yuki; Washio, Naoya; Mifune, Takeshi; Matsuo, Tetsuji

\section{CITATION:}

Sakata, Yuki ...[et al]. Multiple-division subgrid construction for space-time finite integration electromagnetic field computation. International Journal of Applied Electromagnetics and Mechanics 2016, 52(1-2): 617-622

\section{ISSUE DATE:}

2016-12-29

URL:

http://hdl.handle.net/2433/226820

\section{RIGHT:}

(c) 2016 - IOS Press and the authors.; This PDF file can be stored on your computer, it can be printed and saved. It includes the right to post this PDF file on your personal, your institute's, company's or funding agency's website and in their repository. You are not allowed to distribute it for any other purposes (electronic or on paper), and you may not store it in any other repository or in a public retrieval system. 


\title{
Multiple-division subgrid construction for space-time finite integration electromagnetic field computation
}

\author{
Yuki Sakata*, Naoya Washio, Takeshi Mifune and Tetsuji Matsuo \\ Department of Electrical Engineering, Kyoto University, Kyoto, Japan
}

\begin{abstract}
This study proposes an efficient method of local space-time grid refinement and examines the numerical stability and the accuracy of electromagnetic field analysis using the space-time finite-integration technique (FIT). The proposed subgrid method locally refines the space-time grid with three-division or four-division and is stable without inducing numerical instability even after one million time-steps. The grid construction is modified at the corner part of subgrid connection to improve the computational accuracy. The staircase-type grid relaxes the time-step restriction due to the CFL condition.
\end{abstract}

Keywords: Finite integration technique, space-time grid, multiple-division, subgrid

\section{Introduction}

The accurate electromagnetic field analysis of advanced electronic and optical devices [1] often requires the refinement of computational grid, which causes a large computational cost. Even though the subgridding method [1-4] realizes a local refinement of the Yee grid for the finite-difference timedomain (FDTD) method, this method often has some difficulty with unphysical wave reflections and numerical instabilities caused by the non-conformal grid connection. The finite integration technique (FIT) [4-6] enables to use unstructured spatial grids to achieve adaptive grid constructions. Recently, the space-time FIT $[7,8]$ has been developed, which allows a non-uniform time-step distribution naturally. Using the space-time FIT, Ref. [9] proposed a simple and stable subgrid scheme, where the grid refinement is based on the two-division. Basically, the number of division in constructing the subgrid is not restricted by the space-time FIT. This study proposes a more refined subgrid scheme based on the three- or four-division.

\section{Multiple-division scheme}

The formulation of space-time FIT is described in Refs. [7-9]. The space-time grid refinement with the four-division is depicted in Fig. 1, where red solid lines and blue dashed lines denote the primal grids and its dual grids respectively. The spatial and temporal grid-size of the main grid is $\Delta x$ and $\Delta w=c \Delta t$, whereas those of the subgrid are $\Delta x / 4$ and $\Delta w / 4$. Heptagon faces are used between fine and coarse grids so that the primal grid can be orthogonal to the dual grid by the Lorentzian metric.

\footnotetext{
${ }^{*}$ Corresponding author: Yuki Sakata, Department of Electrical Engineering, Kyoto University, Kyoto, 615-8510, Japan. E-mail: yuki1022@fem.kuee.kyoto-u.ac.jp.
} 


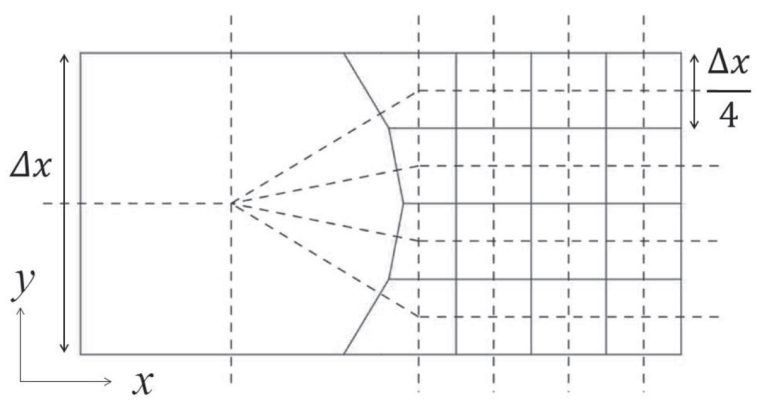

(a) Spatial grid

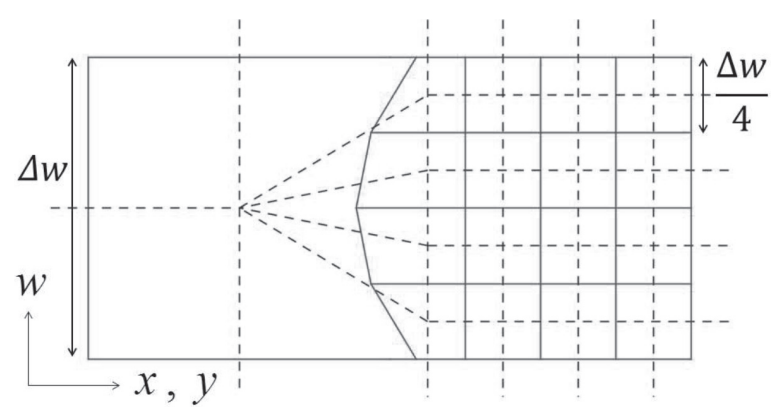

(b) Temporal grid

Fig. 1. Space-time subgrid connection based on four-division.

For simplicity, Fig. 2 illustrates only temporal grid refinement with the 4-division and allocated variables in the connecting part of coarse and fine grids for the analysis of spatially two-dimensional (2D) electromagnetic field having $\left(E_{x}, E_{y}, B_{z}\right)$ components. Variables $e_{x}, e_{y}$ and $h$ are the temporally integrated electromotive and magnetomotive forces respectively, and $d_{x}, d_{y}$ and $b$ are the electric and magnetic fluxes multiplied by $c$. Variables $e_{x}, e_{y}$ and $b$ are allocated to the faces of primal grids and the edges of dual grids are assigned to $d_{x}, d_{y}$ and $h$. The subscripts give spatial indices for the $x$ and $y$ directions, and the superscripts are the temporal indices.

The lengths of edges in Fig. 2 are set as

$$
l_{1}=\Delta x-\delta, l_{2}=l_{1}+\frac{3}{32} \frac{(\Delta w)^{2}}{\Delta x}, l_{3}=l_{2}+\frac{1}{8} \frac{(\Delta w)^{2}}{\Delta x},
$$

where $\delta$ is a free parameter. The variables on the subgrid are updated as

$$
\begin{aligned}
& d_{x, i, j+\frac{1}{2}}^{n+\frac{1}{8}}=d_{x, i, j+\frac{1}{2}}^{n-\frac{1}{8}}+\left(h_{i, j+1}^{n}-h_{i, j}^{n}\right), d_{x, i, j+\frac{3}{2}}^{n+\frac{1}{8}}=d_{x, i, j+\frac{3}{2}}^{n-\frac{1}{8}}+\left(h_{i, j+2}^{n}-h_{i, j+1}^{n}\right) \\
& d_{y, i+\frac{1}{2}, j+1}^{n+\frac{1}{8}}=d_{y, i+\frac{1}{2}, j+1}^{n-\frac{1}{8}}-\left(h_{i+1, j+1}^{n}-h_{i, j+1}^{n}\right), d_{y, i-\frac{1}{2}, j+1}^{n+\frac{1}{8}}=d_{y, i-\frac{1}{2}, j+1}^{n-\frac{1}{8}}-\left(h_{i, j+1}^{n}-h_{i-1, j+1}^{n}\right) \\
& e_{x, i, j+\frac{k}{2}}^{n+\frac{1}{8}}=\frac{\Delta w}{4} d_{x, i, j+\frac{k}{2}}^{n+\frac{1}{8}}(k=1,3), e_{y, i \pm \frac{1}{2}, j+1}^{n+\frac{1}{8}}=\frac{\left(l_{1}+l_{2}\right) \Delta w}{8 \Delta x} d_{y, i \pm \frac{1}{2}, j+1}^{n+\frac{1}{8}} \\
& b_{i, j+1}^{n+\frac{1}{4}}=b_{i, j+1}^{n}+e_{x, i, j+\frac{3}{2}}^{n+\frac{1}{8}}-e_{x, i, j+\frac{1}{2}}^{n+\frac{1}{8}}+e_{y, i-\frac{1}{2}, j+1}^{n+\frac{1}{8}}-e_{y, i+\frac{1}{2}, j+1}^{n+\frac{1}{8}}, \\
& h_{i, j+1}^{n+\frac{1}{4}}=\frac{\Delta w}{4 l_{2} \Delta x} b_{i, j+1}^{n+\frac{1}{4}} .
\end{aligned}
$$

From similar equations to Eqs (2)-(5), $h_{i, j+1}^{n+\frac{k}{4}}(k=2,3,4)$ is obtained, setting $h_{i, j}^{n}$ to 0 after $n+1 / 4$.

On the main grid, the time-marching scheme is given as

$$
d_{x, i, j-\frac{1}{2}}^{n+\frac{1}{2}}=d_{x, i, j-\frac{1}{2}}^{n-\frac{1}{2}}+\left(h_{i, j}^{n}-h_{i, j-1}^{n}\right), d_{y, i \pm \frac{1}{2}, j}^{n+\frac{1}{2}}=d_{y, i \pm \frac{1}{2}, j}^{n-\frac{1}{2}} \mp\left(h_{i, j}^{n}-h_{i \pm 1, j}^{n}\right)
$$




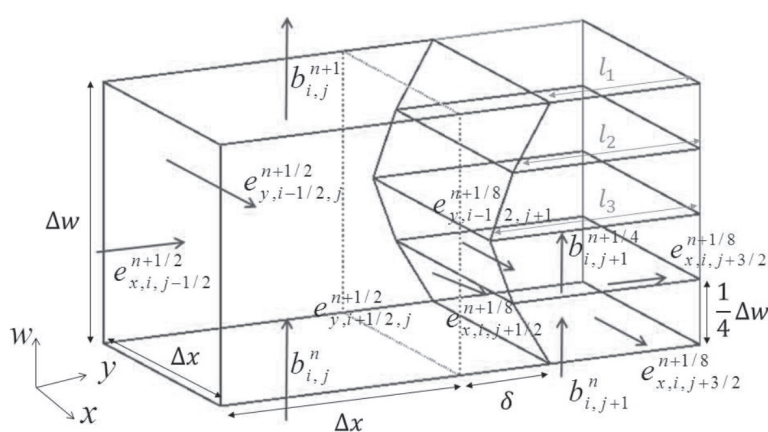

(a) Primal grid

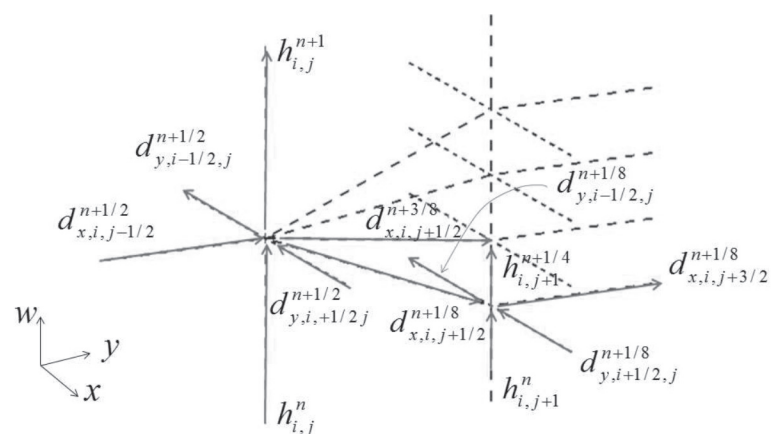

(b) Dual grid

Fig. 2. Variables at the subgrid connection.

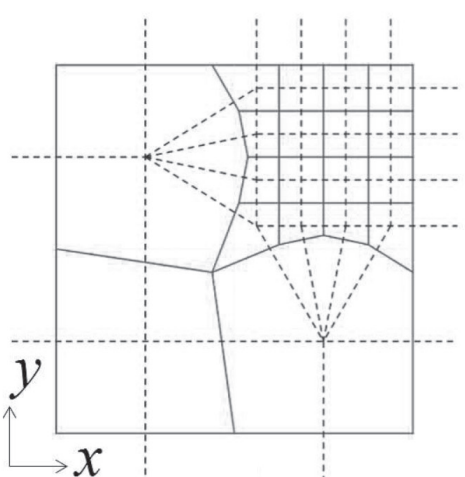

(a)
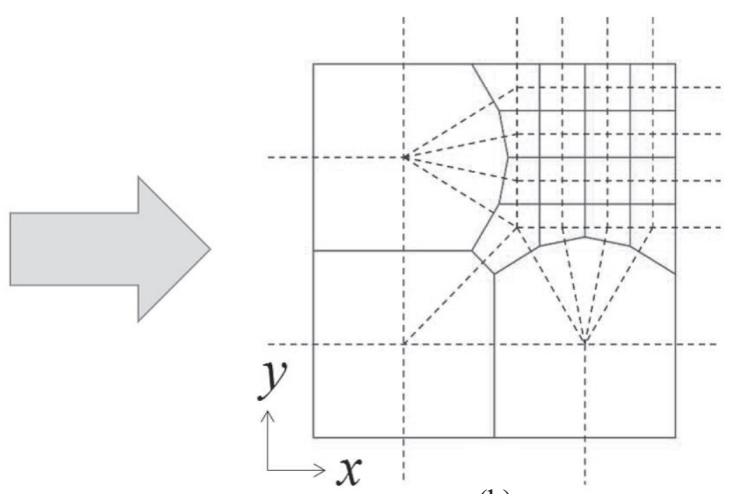

(b)

Fig. 3. Corner modification.

$$
\begin{aligned}
& e_{x, i, j-\frac{1}{2}}^{n+\frac{1}{2}}=\Delta w d_{x, i, j-\frac{1}{2}}^{n+\frac{1}{2}}, e_{y, i \pm \frac{1}{2}, j}^{n+\frac{1}{2}}=\frac{\left(8 \Delta x-l_{1}-2 l_{2}-l_{3}\right) \Delta w}{4 \Delta x} d_{y, i \pm \frac{1}{2}, j}^{n+\frac{1}{2}}, \\
& b_{i, j}^{n+1}=b_{i, j+1}^{n}+e_{x, i, j+\frac{1}{2}}^{n+\frac{1}{8}}+e_{x, i, j+\frac{1}{2}}^{n+\frac{3}{8}}+e_{x, i, j+\frac{1}{2}}^{n+\frac{5}{8}}+e_{x, i, j+\frac{1}{2}}^{n+\frac{7}{8}}-e_{x, i, j-\frac{1}{2}}^{n+\frac{1}{2}}+e_{y, i-\frac{1}{2}, j}^{n+\frac{1}{2}}-e_{y, i+\frac{1}{2}, j}^{n+\frac{1}{2}}, \\
& h_{i, j}^{n+1}=\frac{\Delta w}{\Delta x\left(2 \Delta x-l_{1}\right)} b_{i, j}^{n+1} .
\end{aligned}
$$

\section{Analysis of electromagnetic field}

Whereas the spatially 2D field consisting of $\left(E_{x}, E_{y}, B_{z}\right)$ is described on the faces of primal grids $S_{p}$ and the edges of dual grids $l_{d}$, the faces of dual grids $S_{d}$ and the edges of primal grids $l_{p}$ are used to describe the field mode having $\left(B_{x}, B_{y}, E_{z}\right)$ components. Several issues depending on the two kinds of field modes are discussed in this section. 


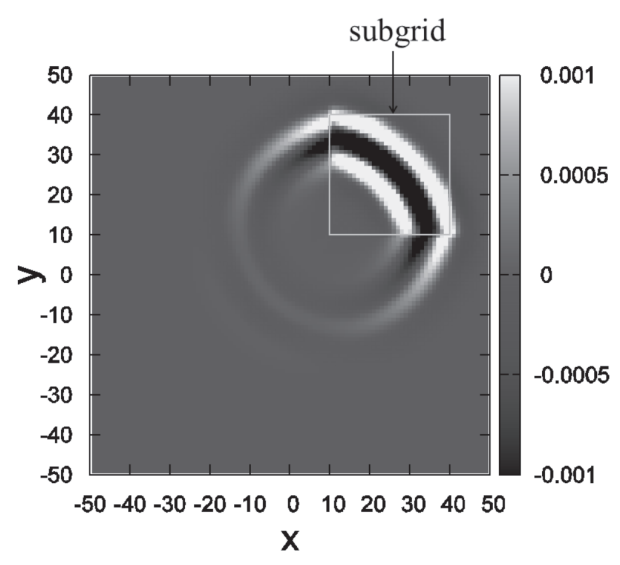

(a) Without corner modification

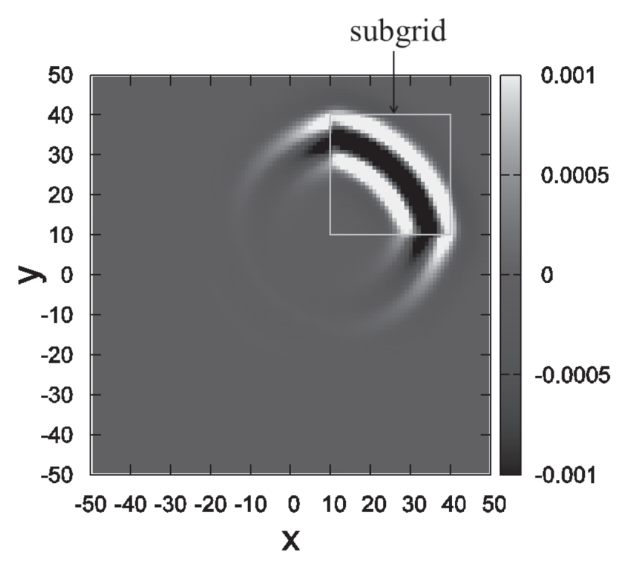

(b) With corner modification

Fig. 4. Discrepancy of $B_{z}$ relative to $B_{z}$ obtained the FDTD method.

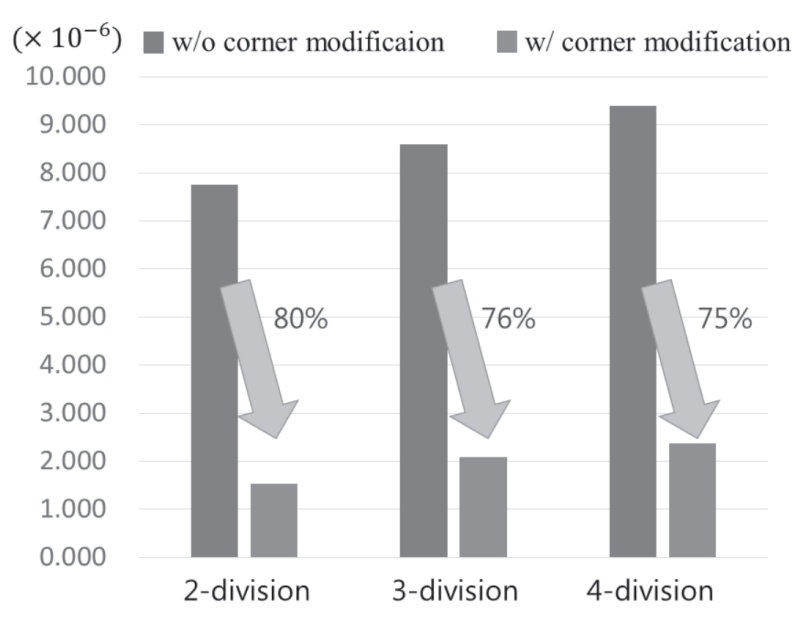

Fig. 5. Average discrepancy of $B_{z}$ from the FDTD method.

\section{1. $\left(E_{x}, E_{y}, B_{z}\right)$ mode}

\subsubsection{Corner modification}

One of the reasons of unphysical wave reflection is the non-orthogonality between the primal and dual grids. A rough connection to the subgrid may yield non-orthogonal intersections of the primal and dual grids at the corner of subgrid, as shown in Fig. 3(a). The corner connection can be modified as in Fig. 3(b) so as for the primal grid to be orthogonal to the dual grid in order to improve the computational accuracy.

\subsubsection{Numerical examination}

For simplicity, the permittivity and the permeability are set to unity by normalization. The analysis domain is given by $-50 \leqslant x \leqslant 50$ and $-50 \leqslant y \leqslant 50$ and the subgrid is located in $10 \leqslant x \leqslant 40$ and $10 \leqslant y \leqslant 40$. The initial conditions are given by $E_{x}=E_{y}=0$ and $B_{z}=\exp \left(-\frac{x^{2}+y^{2}}{25}\right)$. The periodic boundary condition is imposed. The spatial step $\Delta x$ is set to unity by normalization. Figure 4(a) 

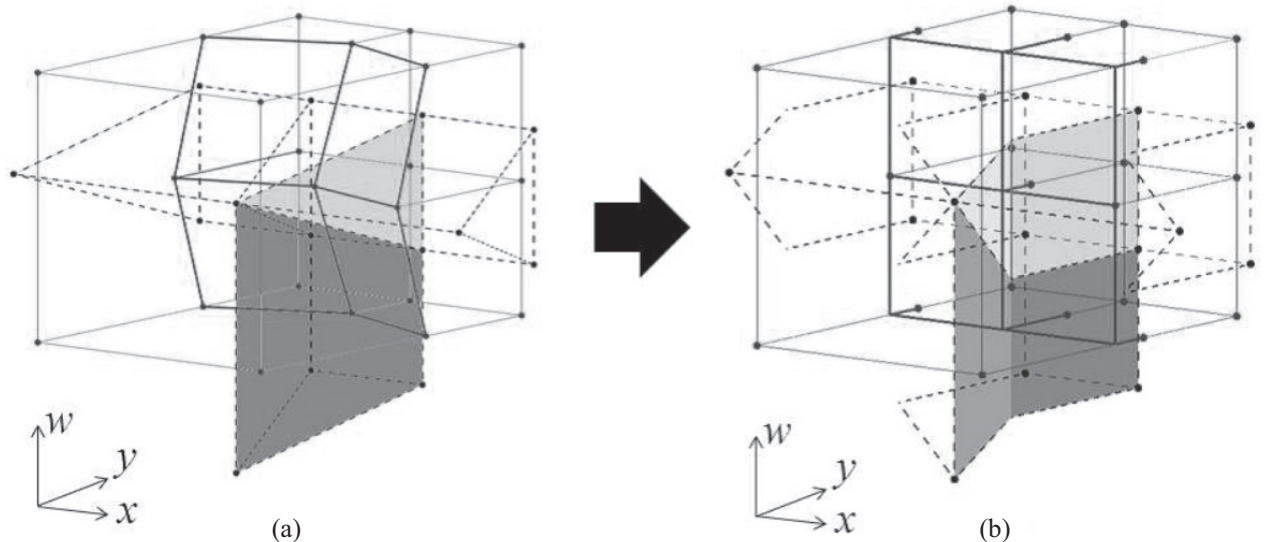

Fig. 6. Transformation of edges from (a) straight-type to (b) staircase-type.

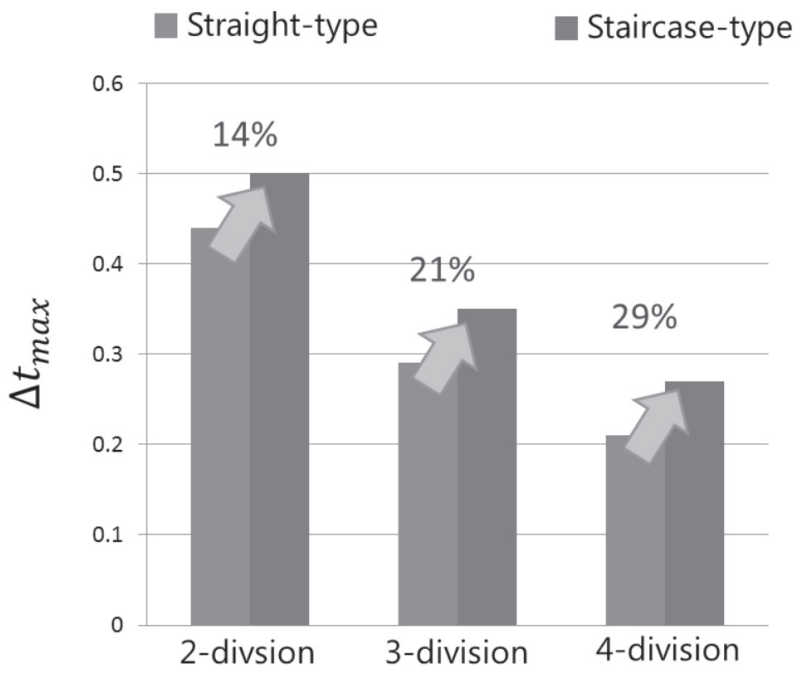

Fig. 7. Maximum time-step.

illustrates the discrepancy of $B_{z}$ between $B_{z}$ yielded by the FDTD method and space-time FIT using 4-division subgrid without the corner modification at $150 \Delta w$ with $\Delta w=0.25$. The discrepancy in the domain $x \leqslant 10$ and $y \leqslant 10$ is due to the unphysical wave reflection at the subgrid connection whereas the discrepancy in the domain $x \geqslant 10$ and $y \geqslant 10$ is caused by the numerical dispersion. Figure 4(b) displays the discrepancy $\Delta B_{z}$ with the corner modification. Figure 5 shows the effect of the corner modification depending on the number of subgrid division by comparing the average of $\left|\Delta B_{z}\right|$ in the domain $x \leqslant 10$ and $y \leqslant 10$. The corner modification reduces the unphysical wave reflection.

\section{2. $\left(B_{x}, B_{y}, E_{z}\right)$ mode}

\subsubsection{The transformation of edges}

The temporal spacing in the dual grid is nonuniform at the subgrid connection. As a result, there are subgrid faces that are relatively wide along the temporal direction compared with the spatial direction. 
Accordingly, the time-step restriction due to the Courant-Friedrichs-Lewy (CFL) condition [3] becomes strong. The transformation of edges without node positions as shown in Fig. 6 enables the width of grids to be roughly uniform. The space-time grids shown in Figs 6(a) and (b) are called straight-type and staircase-type hereafter.

\subsubsection{Numerical examination}

The analyzed domain geometry is similar to that in Subsection 3.1.2. The initial conditions are set as $B_{x}=B_{y}=0$ and $D_{z}=\exp \left(-\frac{x^{2}+y^{2}}{25}\right)$. Figure 7 compares the maximum time-step $\Delta t_{\text {max }}$ that does not induce numerical instability using the straight- and staircase-type grids. The staircase-type grid relaxes the restriction of CFL condition allowing larger time-step than the straight-type.

\section{Conclusion}

The proposed method can increase the number of division in constructing the subgrid while maintaining numerical stability. Instability is not observed even after one million time-steps calculation. The unphysical wave reflection in $\left(E_{x}, E_{y}, B_{z}\right)$ mode is reduced by using the corner modification at the subgrid connection. The staircase-type subgrid connection increases the maximum time-step in the electromagnetic field analysis for $\left(B_{x}, B_{y}, E_{z}\right)$ mode.

\section{References}

[1] Y. Hao and R. Mittra, FDTD Modeling of Metamaterials: Theory and Applications, Artech House, 2008.

[2] S.S. Zivanovic, K.S. Yee and K.K. Mei, A Subgridding Method for The Time-Domain Finite-Difference Method to Solve Maxwell's Equations, IEEE Microwave Theory and Tech 39 (1991), 471-479.

[3] A. Taflove and S.C. Hagness, Computational Electrodynamics, The Finite Difference in Time Domain Method, Artech House, Boston, 2005.

[4] P. Thoma and T. Weiland, A Consistent Subgridding Scheme for The Finite Difference Time Domain Method, Int. J. Numer. Model. 9 (1996), 359-374.

[5] I.E. Lager, E. Tonti, A.T. de Hoop, G. Mur and M. Marrone, Finite Formulation and Domain-Integrated Field Relations in Electromagnetics - A Synthesis, IEEE Trans. Magn. 39 (2003), 1199-1202.

[6] T. Weiland, Time Domain Electromagnetic Field Computation with Finite Difference Methods, Int. J. Numer. Model. 9 (1996), 295-319.

[7] T. Matsuo, Electromagnetic Field Computation Using Space-Time Grid and Finite Integration Method, IEEE Trans. Magn. 46 (2010), 3214-3244.

[8] T. Matsuo, Space-Time Finite Integration Method for Electromagnetic Field Computation, IEEE Trans. Magn. 47 (2011), 1530-1533.

[9] T. Matsuo, T. Shimoi, J. Kawahara and T. Mifune, A Simple Subgrid Scheme Using Space-Time Finite Integration Method, IEEE Trans. Magn. 51 (2015), 7201904. 